\title{
JNFECTIOUS DISEASES
}

\section{FIGURE 7}

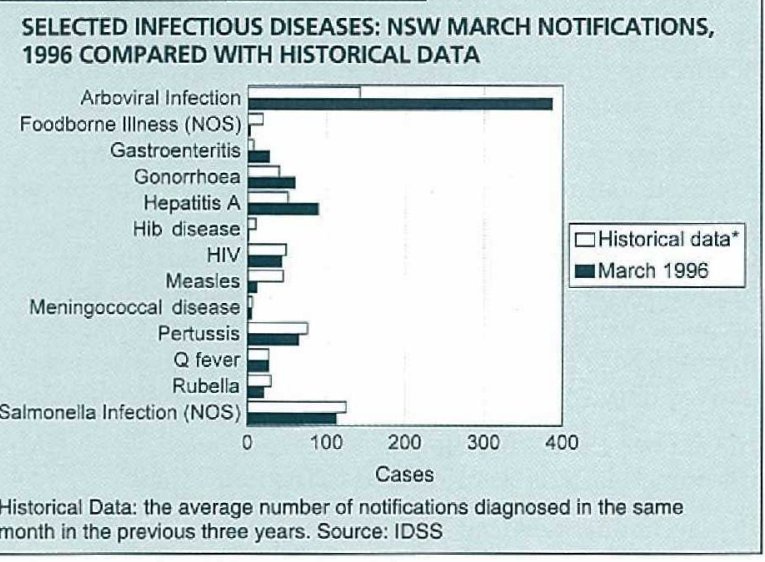

\section{FIGURE 8}

SELECTED INFECTIOUS DISEASES: NSW FEBRUARY NOTIFICATIONS, 1996 COMPARED WITH HISTORICAL DATA

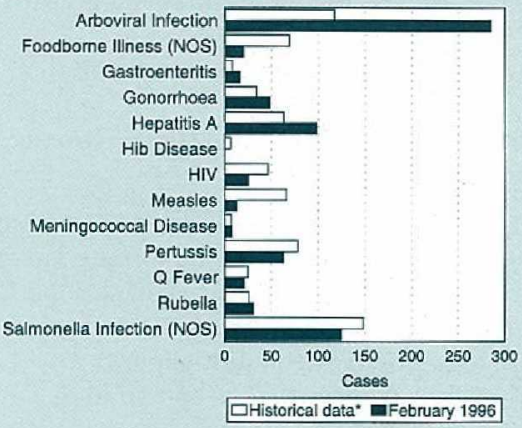

"Historical Data: the average number of notifications diagnosed in the same month in the previous three years. Source: IDSS

Erratum

Figure 8 reproduces Figure 2 from the April Public Health Bulletin. Due to a production error it was difficult to differentiate the historical data from that for February 1996.

\section{FIGURE 9}

ARBOVIRAL INFECTION NOTIFICATIONS: NSW 1994-1996, BY DATE OF TEST FOR DATA RECEIVED BY APRIL 30, 1996

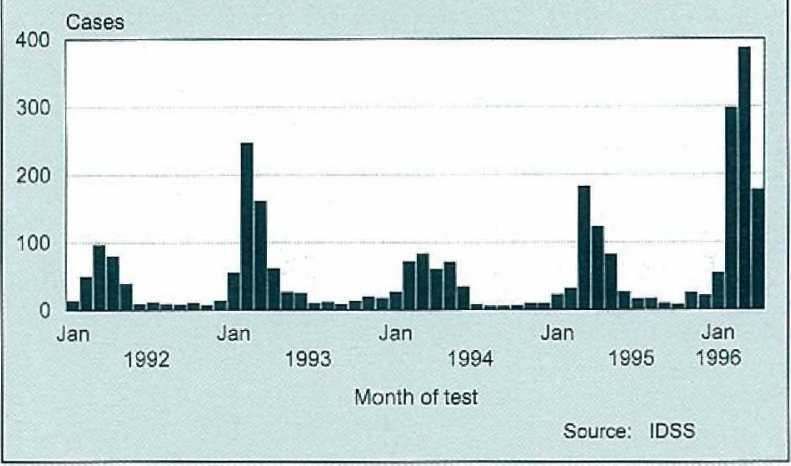

\section{NOTIFICATION TRENDS}

There were more notifications of arboviral infection, gonorrhoea and hepatitis A than expected during March 1996 (Figure 7). Early returns for April suggest that arboviral cases are on the decline across the State after the March peak (Figure 9). Similarly, Statewide data suggest that hepatitis A notifications are declining, at least in Eastern Sydney (Figure 10), where numbers did not reach the 1991 epidemic levels. Early public health intervention, including education on how to reduce exposure, liberal use of immunoglobulin among contacts, and encouragement of vaccination among high-risk groups, may have helped to reduce the numbers of cases.

A second outbreak of hepatitis A has emerged, in the Shoalhaven district of the Illawarra Area. Fifty-nine cases have been reported since February 1996. The Illawarra Public Health Unit (PHU) investigation to date has identified no common source, and indicates that person-toperson transmission is the most likely mode of spread. The PHU has initiated several interventions including active case surveillance, working with local doctors to ensure that contacts of cases are offered immunoglobulin to prevent disease, and educating general practitioners, laboratories, schools, child care centres, patients and contacts, and the general population (through the media) about the disease and its prevention.

\section{INFLUENZA SURVEILLANCE}

Influenza activity appears to be at the same level, or at a slightly lower level, than for the same period in the previous few years.

Reports of influenza-like-illness (ILI) from the NSW Sentinel GP Surveillance Scheme are being received through six PHUs from more than 50 doctors carrying out approximately 7,000 consultations per week. Figure 11 shows that the State average consultation rate for ILI during the first half of May was similar to the average for the previous few years. Western Sydney had the highest consultation rate at 2 per cent.

School absentee rates are being monitored from 10 schools with a total of about 9,000 students, through six PHUs.

Figure 12 shows that the average absentee rate during May was similar to the average for this time of year. The high rates during March were due to causes other than infectious diseases.

Reports from Sydney laboratories indicate for the year to date a small number of cases of influenza A (12 serological, 7 virological diagnoses), and fewer of influenza B (3 serological diagnoses). However, respiratory syncytial virus (RSV) is by far the most commonly diagnosed respiratory virus, and RSV infection is being reported by some laboratories in higher numbers than last year.

EQUINE MORBILLIVIRUS UPDATE: A NATURAL HOST?

Following reports of three human and several equine cases of equine morbillivirus (EMV) infection in Queensland in 1994 and 1995 (NSW Public Health Bulletin, November 1995), Queensland Department of Primary Industry researchers have been searching for a natural reservoir of infection. Since mid-1995, researchers have tested more than 5,000 blood samples from 46 species of domestic and wild animals (including horses, rats, mice, possums, cane toads, rodents, birds, cattle, cats, dogs, pigs, kangaroos, cockroaches, snails, slugs, donkeys and bandicoots) for EMV antibodies. All species had tested negative (except the seven 
horses involved in the original outbreak) until recently, when two species of flying fox from Queensland (the black flying fox and the spectacled flying fox) tested positive. Positive tests indicate exposure to a bat paramyxovirus similar to or the same as EMV. Attempts are being made to isolate the virus, and serological testing of other species of bats and wildlife is continuing. While these findings are intriguing, further data are required to determine whether the bat virus poses a risk to humans.

\section{GONOCOCCAL SURVEILLANCE, NSW, JANUARY-MARCH 1996}

The Gonococcal Reference Laboratory, Microbiology Department, The Prince of Wales Hospital, Randwick, has submitted the following data:

\section{Number of Gonococcal Isolates}

A total of 209 isolates was received in the January-March 1996 quarter, 203 of which remained viable for further examination. This represented an increase on isolates received in the same periods in 1995 (165) and 1994 (144), and was substantially more than the last quarter of 1995 (148).

\begin{tabular}{|lrlr|}
\hline \multicolumn{2}{|c|}{ TABLE 3 } & \\
\hline INFECTED SITES & & \\
\hline MALE PATIENTS & & FEMALE PATIENTS & \\
\hline Urethra & 131 & Endocervix/vagina & 37 \\
Pharynx & 18 & Pharynx & 0 \\
Ano-rectum & 21 & Ano-rectum & 0 \\
Blood & 0 & Blood & 0 \\
Eye & 2 & Eye & 0 \\
Other & 0 & Other & 0 \\
\hline Total & 172 & Total & 37 \\
\hline
\end{tabular}

Isolates were obtained from males and females in the ratio of 4.6:1. For gonococcal isolates from men, rectal isolates comprised 12.2 per cent and pharyngeal isolates $10.5 \mathrm{per}$ cent. All the isolates from females were from the endocervix. There were two ophthalmic infections in this quarter, one in a neonate.

\section{Antibiotic sensitivity patterns}

Penicillins (including penicillin, ampicillin and amoxycillin) The pattern of gonococcal susceptibility of the penicillins remained essentially unchanged in this quarter and indicates that use of penicillin-based treatment regimens (including amoxycillin and ampicillin) would result in a significant proportion of treatment failures. One-third of all gonococci examined were resistant to the penicillins. Sixtyseven of the 203 strains tested were penicillin-resistant, 20 being PPNG (penicillinase-producing $N$. gonorrhoeae) and 47 resistant by chromosomal mechanisms (CMRNG). PPNG were isolated from patients who contracted their infection locally and overseas.

\section{Ceftriaxone}

All isolates examined were sensitive to this injectable antibiotic which has retained its activity against gonococci for many years.

\section{Spectinomycin}

All strains were susceptible in vitro to this injectable antibiotic.

Quinolone group (ciprofloxacin, norfloxacin, enoxacin) Gonococci with low-level resistance to the quinolone group

\section{FIGURE 10}

HEPATITIS A NOTIFICATIONS NSW 1992-1996,

BY DATE OF ONSET FOR DATA RECEIVED BY APRIL 30, 1996

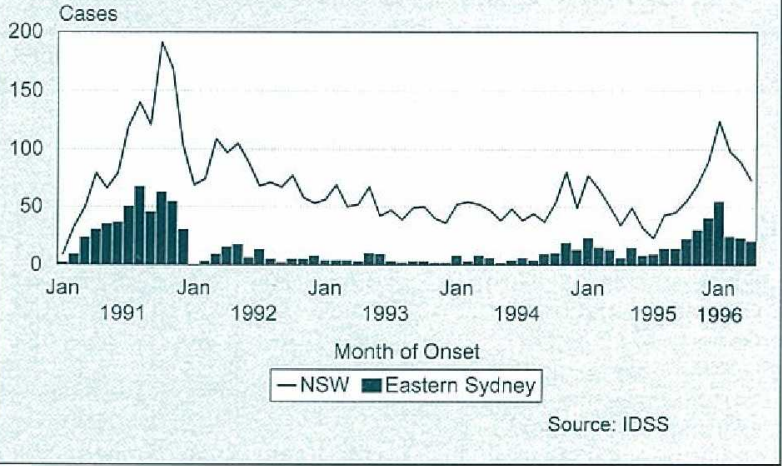

\section{FIGURE 11}

NSW GP SENTINEL SURVEILLANCE -

INFLUENZA-LIKE ILLNESS 1996
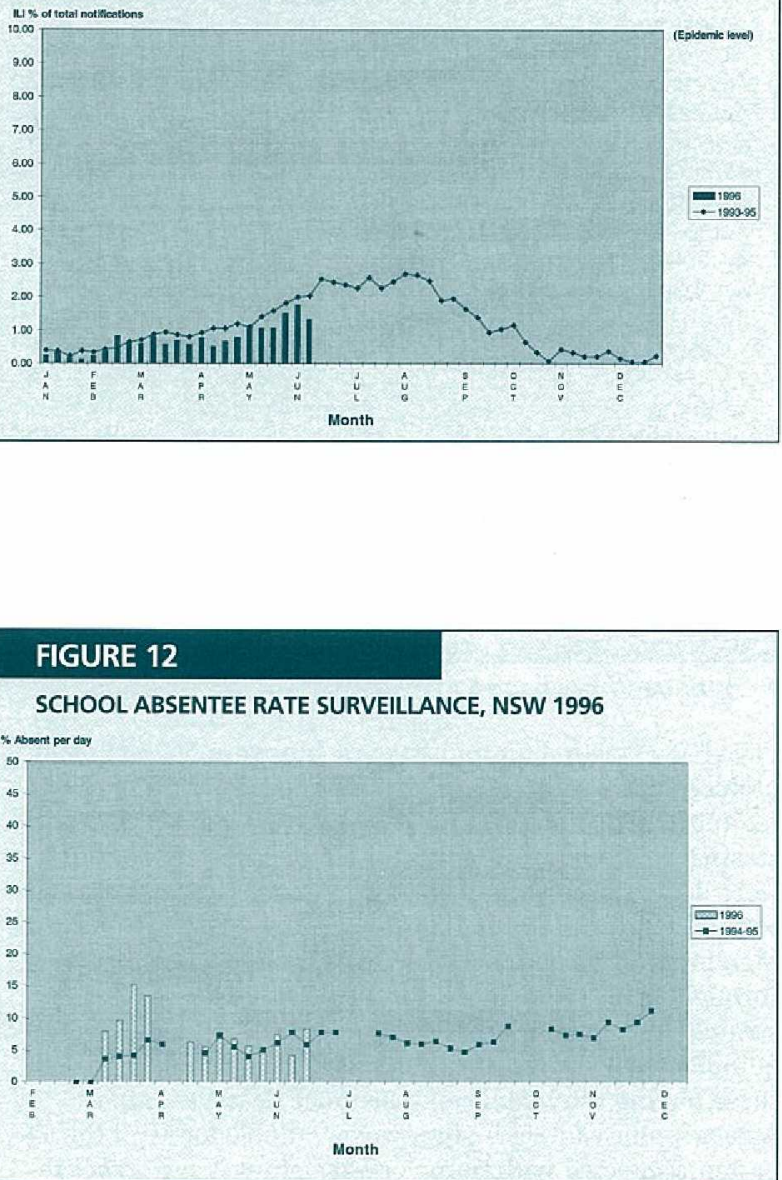


\section{TABLE 4}

INFECTIOUS DISEASE NOTIFICATIONS FOR NSW, 1996 BY MONTH OF ONSET FOR NOTIFICATIONS

RECEIVED BY APRIL 30, 1996

\begin{tabular}{|c|c|c|c|c|c|}
\hline Condition & Jan & Feb & Mar & Apr & Total \\
\hline $\begin{array}{l}\text { Adverse event after } \\
\text { immunisation }\end{array}$ & 9 & 4 & 3 & 2 & \\
\hline $\begin{array}{l}\text { immunisation } \\
\text { AIDS }\end{array}$ & 35 & 23 & 22 & $\begin{array}{r}2 \\
11\end{array}$ & $\begin{array}{l}18 \\
91\end{array}$ \\
\hline Arboviral infection & 51 & 297 & 383 & 179 & 910 \\
\hline Brucellosis & - & 1 & - & - & 1 \\
\hline Cholera & - & - & 1 & - & 1 \\
\hline Foodborne illness (NOS) & 17 & 20 & 8 & 2 & 47 \\
\hline Gastroenteritis (instit.) & 11 & 16 & 27 & - & 54 \\
\hline Gonorrhoea infection & 46 & 48 & 60 & 30 & 184 \\
\hline H. influenzae epiglottitis & - & - & 1 & - & 1 \\
\hline $\mathrm{H}$. influenzae meningitis & 1 & - & - & - & 1 \\
\hline H. influenzae septicaemia & - & - & - & 1 & 1 \\
\hline Hepatitis A - acute viral & 124 & 98 & 89 & 63 & 374 \\
\hline Hepatitis B - acute viral & 7 & - & 3 & 3 & 13 \\
\hline Hepatitis B - chronid/carrier & 68 & 69 & 91 & 85 & 313 \\
\hline Hepatitis B - unspecified & 306 & 331 & 343 & 196 & 1,176 \\
\hline Hepatitis C-acute viral & - & - & 2 & 1 & 3 \\
\hline Hepatitis C - unspecified & 719 & 730 & 645 & 405 & 2,499 \\
\hline Hepatitis D - unspecified & - & 1 & 1 & - & 2 \\
\hline Hepatitis, acute viral (NOS) & 3 & - & - & - & 3 \\
\hline HIV infection & 37 & 23 & 43 & 34 & 137 \\
\hline Hydatid disease & 1 & 2 & 2 & 2 & 7 \\
\hline Legionnaires' disease & 4 & 10 & 8 & 6 & 28 \\
\hline Leprosy & - & - & 1 & - & 1 \\
\hline Leptospirosis & 3 & 3 & 5 & 1 & 12 \\
\hline Listeriosis & 2 & - & - & 1 & 3 \\
\hline Malaria & 22 & 22 & 22 & 14 & 80 \\
\hline Measles & 21 & 13 & 12 & 12 & 58 \\
\hline Meningococcal infection (NOS) & 1 & 3 & 1 & - & 5 \\
\hline Meningococcal meningitis & 6 & 3 & 3 & 5 & 17 \\
\hline Meningococcal septicaemia & 2 & 2 & 1 & 2 & 7 \\
\hline Mumps & 5 & 6 & - & - & 11 \\
\hline Mycobacterial atypical & 36 & 38 & 12 & 5 & 91 \\
\hline Mycobacterial infection (NOS) & 8 & 12 & 9 & 7 & 36 \\
\hline Mycobacterial tuberculosis & 40 & 23 & 19 & 8 & 90 \\
\hline Pertussis & 101 & 68 & 65 & 50 & 284 \\
\hline Q fever & 22 & 21 & 23 & 21 & 87 \\
\hline Rubella & 41 & 31 & 21 & 15 & 108 \\
\hline Salmonella (NOS) & 132 & 126 & 108 & 84 & 450 \\
\hline Syphilis infection & 60 & 65 & 78 & 41 & 244 \\
\hline Typhoid and paratyphoid & 7 & 5 & 5 & - & 17 \\
\hline Vibrio infection (non cholera) & 1 & 1 & - & 1 & 3 \\
\hline
\end{tabular}

\section{Infectious diseases}

\section{Continued from page 51}

have been isolated for many years, but were not a clinical problem if the recommended higher dose treatment regimens (e.g. $500 \mathrm{mg}$ ciprofloxacin) were used. In 1994, strains with higher minimum inhibitory concentrations were detected in Sydney and Port Kembla for which no dose of quinolone antibiotic would be effective. Isolates with this high level quinolone resistance continued to appear throughout 1995 and accounted for 3.5 per cent of the strains examined. Eleven isolates (5.4 per cent) with altered quinolone resistance were detected in this quarter, nine of these having the high level resistance described above. A geographic contact history was available for 10 of the 11 patients infected with quinolone-resistant $N$ gonorrhoeae (QRNG). One locally acquired infection with a low-level resistant strain was recorded. All the other strains were isolated from patients infected in South East Asia. Data from World Health Organization sources indicate that more
TABLE 5

SUMMARY OF NSW INFECTIOUS DISEASE NOTIFICATIONS APRIL 1996

\begin{tabular}{|c|c|c|c|c|}
\hline \multirow[t]{3}{*}{ Condition } & \multicolumn{4}{|c|}{ Number of cases notified } \\
\hline & \multicolumn{2}{|c|}{ Period } & \multicolumn{2}{|c|}{ Cumulative } \\
\hline & $\begin{array}{r}\text { Apr } \\
1995\end{array}$ & $\begin{array}{r}\text { Apr } \\
1996\end{array}$ & $\begin{array}{r}\text { Apr } \\
1995\end{array}$ & $\begin{array}{r}\text { Apr } \\
1996\end{array}$ \\
\hline Adverse reaction & 1 & 2 & 8 & 18 \\
\hline AIDS & 33 & 11 & 158 & 91 \\
\hline Arboviral infection & 121 & 179 & 356 & 910 \\
\hline Brucellosis & - & - & - & 1 \\
\hline Cholera & - & - & - & 1 \\
\hline Diphtheria & - & - & - & - \\
\hline Foodborne illness (NOS) & 28 & 2 & 262 & 47 \\
\hline Gastroenteritis (instit.) & 35 & - & 50 & 54 \\
\hline Gonorrhoea & 30 & 30 & 144 & 184 \\
\hline $\mathrm{H}$ influenzae epiglottitis & 2 & - & 3 & 1 \\
\hline $\mathrm{H}$ influenzae B - meningitis & - & - & 3 & 1 \\
\hline H influenzae B - septicaemia & - & 1 & 3 & 1 \\
\hline $\mathrm{H}$ influenzae infection (NOS) & 1 & - & 2 & - \\
\hline Hepatitis A & 34 & 63 & 227 & 374 \\
\hline Hepatitis B & 369 & 284 & 1,679 & 1,502 \\
\hline Hepatitis C & 563 & 406 & 2,935 & 2,502 \\
\hline Hepatitis D & 2 & - & 8 & 2 \\
\hline Hepatitis, acute viral (NOS) & - & - & - & 3 \\
\hline HIV infection & 34 & 34 & 173 & 137 \\
\hline Hydatid disease & - & 2 & 4 & 7 \\
\hline Legionnaires' disease & 2 & 6 & 36 & 28 \\
\hline Leprosy & - & - & 1 & 1 \\
\hline Leptospirosis & 1 & 1 & 3 & 12 \\
\hline Listeriosis & - & 1 & 7 & 3 \\
\hline Malaria & 10 & 14 & 58 & 80 \\
\hline Measles & 34 & 12 & 257 & 58 \\
\hline Meningococcal meningitis & 2 & 5 & 14 & 17 \\
\hline Meningococcal septicaemia & - & 2 & 7 & 7 \\
\hline Meningococcal infection (NOS) & 3 & - & 9 & 5 \\
\hline Mumps & - & - & 2 & 11 \\
\hline Mycobacterial tuberculosis & 23 & 8 & 155 & 90 \\
\hline Mycobacterial - atypical & 34 & 5 & 183 & 91 \\
\hline Mycobacterial infection (NOS) & 5 & 7 & 17 & 36 \\
\hline Pertussis & 70 & 50 & 297 & 284 \\
\hline Plague & - & - & - & - \\
\hline Poliomyelitis & - & - & - & - \\
\hline Q fever & 12 & 21 & 66 & 87 \\
\hline Rubella & 27 & 15 & 138 & 108 \\
\hline Salmonella infection (NOS) & 204 & 84 & 570 & 450 \\
\hline Syphilis & 68 & 41 & 315 & 244 \\
\hline Tetanus & - & - & - & - \\
\hline Typhoid and paratyphoid & 5 & - & 27 & 17 \\
\hline Typhus & - & - & - & - \\
\hline Viral haemorrhagic fevers & - & - & - & - \\
\hline Yellow fever & - & - & - & - \\
\hline
\end{tabular}

than 25 per cent of isolates in the Philippines, Hong Kong, China, Korea and Japan have altered quinolone resistance. Consideration should be given to the treatment regimen employed for patients who acquire their infection in these regions.

Tetracyclines

The tetracycline group is not recommended for the treatment of gonococcal infection. All of the above agents can be administered as single dose therapy to ensure patient compliance whereas the tetracycline treatment regimens are multiple-dose therapies. A further reason for not using tetracycline-based regimens is the resistance of gonococci to these agents. The most recent examination of tetracycline resistance patterns indicated that about 30 per cent of NSW isolates were resistant. Additionally, a form of high-level plasmid resistance to the tetracyclines has emerged in the 
TABLE 6

INFECTIOUS DISEASE CUMULATIVE NOTIFICATIONS FOR NSW, 1996 BY PUBLIC HEALTH UNIT RECEIVED BY APRIL 30, 1996

\begin{tabular}{|c|c|c|c|c|c|c|c|c|c|c|c|c|c|c|c|c|c|c|}
\hline Condition & CCA & CSA & CW & ESA I & IUN & ILL & NC & ND & NSA & SE & SSA & SW & SWS & WEN & WN & WSA & U/K & Total \\
\hline $\begin{array}{l}\text { AIDS } \\
\text { Arboviral infection }\end{array}$ & $\begin{array}{l}3 \\
8\end{array}$ & $\begin{array}{r}18 \\
3\end{array}$ & 20 & $\begin{array}{r}26 \\
4\end{array}$ & $\begin{array}{r}5 \\
53\end{array}$ & 6 & $\begin{array}{r}4 \\
299\end{array}$ & 238 & $\begin{array}{l}15 \\
15\end{array}$ & $\overline{9}$ & $\begin{array}{l}2 \\
3\end{array}$ & $\begin{array}{r}1 \\
86\end{array}$ & $\begin{array}{r}12 \\
7\end{array}$ & $\underline{4}$ & 154 & $\begin{array}{l}1 \\
5\end{array}$ & $\overline{-}$ & $\begin{array}{r}91 \\
910\end{array}$ \\
\hline ritis ( & - & 9 & - & - & 18 & - & - & 1 & - & - & - & - & 1 & 8. & 1 & $\frac{1}{16}$ & - & \\
\hline $\begin{array}{l}\mathrm{G} \\
\mathrm{H} s\end{array}$ & $\begin{array}{l}3 \\
1\end{array}$ & 16 & $\frac{3}{-}$ & $\begin{array}{r}90 \\
7\end{array}$ & 2 & $\underline{2}$ & $\begin{array}{l}7 \\
1\end{array}$ & - & 11 & 3 & $\begin{array}{r}10 \\
1\end{array}$ & - & $\begin{array}{l}5 \\
1\end{array}$ & & $\begin{array}{r}13 \\
1\end{array}$ & $\begin{array}{l}9 \\
1\end{array}$ & $\overline{-}$ & $\begin{array}{r}184 \\
13\end{array}$ \\
\hline & 15 & & 6 & 98 & & & 7 & 1 & & $\bar{c}$ & 17 & $\overline{0}$ & 41 & 6 & 3 & 118 & - & 313 \\
\hline & $\begin{array}{r}10 \\
1\end{array}$ & $\begin{array}{r}146 \\
1\end{array}$ & 2 & 40 & 30 & 32 & 23 & 6 & 17: & 6 & 210 & 9 & 354 & 7 & 10 & $\begin{array}{r}118 \\
1\end{array}$ & $\overline{-}$ & $\begin{array}{r}1,176 \\
3\end{array}$ \\
\hline C- unspecified & 104 & 237 & 76 & 325 & 164 & 154 & $\begin{array}{r}243 \\
2\end{array}$ & 61 & 195 & 44 & 150 & 56 & 298 & 119 & 29 & 243 & - & 2,499 \\
\hline T (NOS) & $\overline{1}$ & $1 \overline{7}$ & $\begin{array}{l}1 \\
1 \\
1\end{array}$ & $\begin{array}{r}1 \\
46\end{array}$ & $\overline{5}$ & $\overline{3}$ & $\overline{1}$ & $\overline{1}$ & $\overline{9}$ & $\overline{-}$ & $\overline{2}$ & $\overline{1}$ & $\overline{9}$ & 4 & $\overline{-}$ & ${ }_{11}^{1}$ & $2 \overline{6}$ & $\begin{array}{r}3 \\
137\end{array}$ \\
\hline is' disease & $\overline{2}$ & $\begin{array}{l}1 \\
2\end{array}$ & 2 & $\overline{-}$ & $\begin{array}{l}1 \\
3\end{array}$ & $\overline{1}$ & $\begin{array}{l}1 \\
1\end{array}$ & $\overline{-}$ & $\overline{2}$ & $\overline{3}$ & $\overline{1}$ & - & $\begin{array}{l}1 \\
5\end{array}$ & 1 & - & 7 & - & 28 \\
\hline & & - & 1 & $\overline{-}$ & 4 & $\overline{-}$ & $\overline{4}$ & $\overline{2}$ & 1 & $\overline{-}$ & - & - & $\overline{1}$ & & $\begin{array}{l}- \\
\end{array}$ & $\begin{array}{c}- \\
-\end{array}$ & $\overline{-}$ & 12 \\
\hline & 1 & 10 & 2 & 8 & 9 & 5 & 4 & 4 & 11 & 2 & 5 & 2 & 5 & 3 & 1 & 8 & - & 80 \\
\hline & 1 & 1 & - & - & 6 & 2 & 2 & - & - & 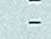 & 1 & - & $\overline{-}$ & $\overline{1}$ & 2 & 1 & & 17 \\
\hline & 7 & 10 & $\begin{array}{l}3 \\
1\end{array}$ & 13 & $\begin{array}{l}1 \\
2\end{array}$ & - & 9 & $\overline{2}$ & $1 \overline{3}$ & $\overline{-}$ & $\begin{array}{l}1 \\
6\end{array}$ & $\begin{array}{l}1 \\
1\end{array}$ & $\begin{array}{r}1 \\
10\end{array}$ & 5 & $\overline{1}$ & $1 \overline{1}$ & - & \\
\hline $\begin{array}{l}\text { Myco } \\
\text { Myco }\end{array}$ & $\begin{array}{l}2 \\
6\end{array}$ & $\begin{array}{l}5 \\
5\end{array}$ & 1 & $\begin{array}{l}1 \\
8\end{array}$ & $\begin{array}{l}5 \\
2\end{array}$ & $\overline{-}$ & $\underline{4}$ & $\begin{array}{l}1 \\
1\end{array}$ & $\begin{array}{r}5 \\
15\end{array}$ & $\overline{-}$ & $\begin{array}{r}5 \\
12\end{array}$ & $\overline{-}$ & ${ }_{21}^{1}$ & $\frac{1}{2}$ & 1 & $\begin{array}{r}5 \\
17\end{array}$ & $\overline{-}$ & $\begin{array}{l}36 \\
90\end{array}$ \\
\hline & & & & & 2 & - & 9 & 23 & & 1 & & 6 & & $=$ & 38 & - & - & 87 \\
\hline $\begin{array}{l}\text { Syphilis infection } \\
\text { Vibrio infection (non cholera) }\end{array}$ & & 27 & 4 & $\begin{array}{r}44 \\
1\end{array}$ & 11 & 2 & 14 & 20 & 21 & 2 & 9 & 1 & $\begin{array}{r}34 \\
2\end{array}$ & 2 & 27 & 23 & $\bar{z}$ & 244 \\
\hline
\end{tabular}

\section{TABLE 7}

VACCINE PREVENTABLE AND RELATED CONDITIONS, CUMULATIVE NOTIFICATIONS FOR NSW, 1996 BY PUBLIC HEALTH UNIT, RECEIVED BY APRIL 30, 1996

\begin{tabular}{|c|c|c|c|c|c|c|c|c|c|c|c|c|c|c|c|c|c|}
\hline Condition & CCA & CSA & CW & ESA & HUN & ILL & NC & ND & NSA & SE & SSA & SW & SWS & WEN & WN & WSA & Total \\
\hline $\begin{array}{l}\text { Adverse event after } \\
\text { immunisation } \\
\text { H. influenzae epiglottitis } \\
\text { H. influenzae meningitis } \\
\text { H. influenzae septicaemia } \\
\text { Measles } \\
\text { Mumps } \\
\text { Pertussis } \\
\text { Rubella }\end{array}$ & $\begin{array}{l}- \\
\overline{-} \\
\overline{1} \\
\overline{4} \\
-\end{array}$ & $\begin{array}{r}- \\
\overline{-} \\
\overline{2} \\
1 \\
10 \\
28\end{array}$ & $\begin{array}{l}\overline{2} \\
\overline{1} \\
\overline{4} \\
\overline{4} \\
1\end{array}$ & $\frac{\overline{1}}{\overline{-}}$ & $\begin{array}{r}- \\
\overline{-} \\
\overline{1} \\
1 \\
2 \\
34 \\
-\end{array}$ & $\begin{array}{r}\overline{-} \\
\overline{-} \\
\overline{5} \\
\overline{19} \\
9\end{array}$ & $\begin{array}{l}\frac{2}{-} \\
\overline{2} \\
\overline{2} \\
\overline{2} \\
2\end{array}$ & $\begin{array}{r}\overline{-} \\
\overline{2} \\
\overline{21} \\
-\end{array}$ & $\begin{array}{l}- \\
\overline{-} \\
\overline{1} \\
5 \\
33 \\
-\end{array}$ & $\begin{array}{r}\frac{8}{-} \\
\overline{3} \\
\overline{3} \\
15 \\
2\end{array}$ & $\begin{array}{l}1 \\
- \\
- \\
\overline{5} \\
1 \\
9 \\
6\end{array}$ & $\begin{array}{r}1 \\
- \\
- \\
5 \\
1 \\
24 \\
-\end{array}$ & $\begin{array}{r}1 \\
\overline{-} \\
\overline{7} \\
1 \\
13 \\
-\end{array}$ & $\begin{array}{r}\frac{1}{-} \\
\overline{-} \\
\overline{2} \\
\overline{5} \\
16\end{array}$ & $\begin{array}{l}- \\
\overline{-} \\
\overline{4} \\
\overline{4} \\
-\end{array}$ & $\begin{array}{l}2 \\
\overline{-} \\
\overline{12} \\
\overline{37} \\
43\end{array}$ & $\begin{array}{r}18 \\
1 \\
1 \\
1 \\
58 \\
11 \\
284 \\
108\end{array}$ \\
\hline
\end{tabular}

\section{TABLE 8}

FOODBORNE INFECTIOUS DISEASE CUMULATIVE NOTIFICATIONS FOR NSW, 1996

BY PUBLIC HEALTH UNIT, RECEIVED BY APRIL 30, 1996

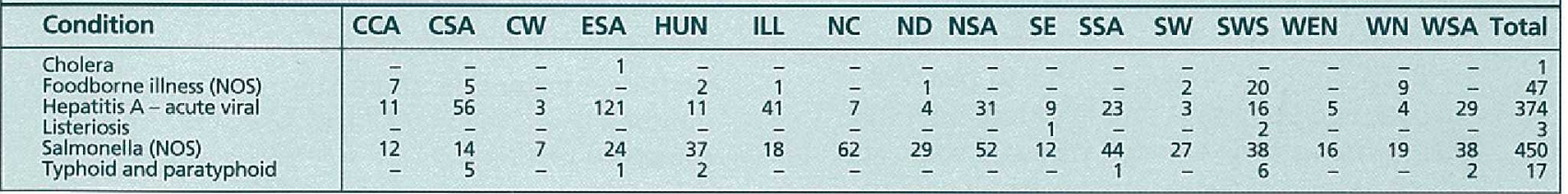

past decade. Isolates possessing this plasmid are known as tetracycline-resistant $N$ gonorrhoeae (TRNG). Thirteen TRNG were detected in this quarter (6.4 per cent of all isolates). Again, most of the strains were acquired overseas but two TRNG infections were acquired locally. Eleven TRNG were detected in the December quarter.

\section{Editorial Note}

The trends reported by the National Gonorrhoea

Surveillance Scheme (NGSS) are reflected in NSW laboratory notifications of gonorrhoea, which were higher during the January-March quarter this year (154) than the previous quarter (115) and the same period last year (114). As previously discussed in the Bulletin, this may be due to a cycle in which gonorrhoea incidence peaks approximately every four years. NSW PHUs received fewer gonnorrhoea notifications than reported by NGSS, however, suggesting under-reporting by laboratories.

\section{Abbreviations used in this Bulletin:}

CSA Central Sydney Health Area, SSA Southern Sydney Health Area, ESA Eastern Sydney Health Area, SWS South Western Sydney Health Area, WSA Western Sydney Health Area, WEN Wentworth Health Area, NSA Northern Sydney Health Area, CCA Central Coast Health Area, ILL IIlawarra Health Area, HUN Hunter Health Area, NC North Coast Public Health Unit, ND Northern District Public Health Unit WN Western New South Wales Public Health Unit, CW Central West Public Health Unit, SW South West Public Health Unit, SE South East Public Health Unit, OTH Interstate/Overseas, U/K Unknown, NOS Not Otherwise Stated.

Please note that the data contained in this Bulletin are provisional and subject to change because of late reports or changes in case classification. Data are tabulated where possible by area of residence and by the disease onset date and not simply the date of notification or receipt of such notification. 